\section{Review: limited evidence to support pharmacological therapy for amphetamine withdrawal}

\section{QUESTION}

Question: Is pharmacological therapy, alone or in combination with psychosocial treatment, effective for amphetamine withdrawal?

Outcomes: Discontinuation rates, average global state score (eg, Clinical Global Impression), average score on withdrawal symptomatology assessments (eg, Amphetamine Withdrawal Questionnaire), average score in craving rating scales (eg, Questionnaire for Evaluating Cocaine Craving and Related Responses) and patient satisfaction (measured by type and number of adverse events).

\section{METHODS}

Design: Systematic review with meta-analysis.

Data sources: The Cochrane Central Register of Controlled Trials, MEDLINE, PsycINFO and CINAHL (searched from inception to 1 May 2008). Reference lists of identified studies and conference proceedings were hand searched.

Study selection and analysis: Two independent reviewers appraised the studies and selected randomised controlled trials comparing any kind of pharmacological treatment, alone or in combination with a psychosocial treatment, placebo or any psychosocial treatment for amphetamine withdrawal. Studies of other drugs in addition to amphetamine were included provided $>50 \%$ of participants were withdrawing from amphetamine and there was separate reporting for amphetamine. Heterogeneity was assessed using $\chi^{2}$ tests. Relative risk and number needed to treat were calculated for dichotomous outcomes and weighted mean difference (WMD) calculated for continuous data using the fixed effects model.

\section{MAIN RESULTS}

Four studies ( $\mathrm{n}=125 ; 88 \%$ male) met the inclusion criteria. Two studies compared amineptine (300 mg daily) with placebo in inpatients at a drug dependence centre who met DSM-IV criteria for amphetamine withdrawal, and two studies compared mirtazapine (15-60 mg, titrated to need) with placebo in participants who also had an Amphetamine Withdrawal Questionnaire score of $\geqslant 10$. Treatment duration was 2 weeks in all studies. Any active treatment significantly decreased the risk of discontinuation compared with placebo (four randomised controlled trials (RCTs), 125 participants; relative risk 0.52 , 95\% confidence interval (CI) 0.29 to 0.94 ; $\mathrm{p}=0.03)$. Amineptine significantly improved global state on the Clinical Global Impressions scale compared with placebo (2 RCTs; WMD -0.49 , 95\% CI -0.80 to -0.17 ; $p=0.002$ ) but mirtazapine did not (1 RCT; WMD 0.30, 95\% CI -0.22 to $0.82 ; p=0.25)$. One amineptine study and one mirtazapine study found no significant effect of treatment on average withdrawal symptom scores (2 RCTs, 74 participants; SMD $-0.08,95 \%$ CI -0.54 to 0.38$)$. One amineptine study found no significant effect on average craving score compared with placebo.

\section{CONCLUSIONS}

There is limited evidence of effect for treatments of amphetamine withdrawal. Amineptine reduced treatment discontinuation rates and improved clinical presentation compared with placebo but had no effect on reducing withdrawal symptoms or craving, and is currently not available for use. There is little evidence of any benefit of mirtazepine.

\section{ABSTRACTED FROM}

Shoptaw SJ, Kao U, Heinzerling K, et al. Treatment for amphetamine withdrawal. Cochrane Database Syst Rev 2009; 2:CD003021.

Correspondence to: Steven J Shoptaw, Department of Family Medicine, University of California, 10880 Wilshire Boulevard, Suite 540, Los Angeles, CA 90024, USA; sshoptaw@mednet.ucla.edu

Source of funding: UCLA Integrated Substance Abuse Programs, USA. worldwide, estimates suggest that there may be almost 25 million users of these drugs. Unquestionably, some users become seriously dependent on amphetamines in the sense that they experience strong, and sometimes overpowering, urges to use these drugs, and this is reflected in an impaired capacity to control their drug taking. There continues to be considerable uncertainty about whether amphetamines (in this review, amphetamine, dextroamphetamine and methamphetamine) should be regarded as having a true withdrawal syndrome (ie, a predictable set of symptoms which are reliably found across users). Nonetheless, many users report symptoms which are clinically severe in that they may lead to subjective distress, premature termination of treatment, relapse to further drug use, and suicidal ideation and attempts. For these reasons, any effective pharmacotherapy for amphetamine withdrawal would have great clinical and public health value.

This review provides a well written and thoughtful review of the issue and its context. The review is seriously limited by the paucity of evidence that is allowed within the very strict confines of the Cochrane Collaboration: only four studies involving a total of 125 participants met the criteria for inclusion. Two studies compared amineptine (an atypical tricyclic antidepressant which has pharmacological effects similar to those of amphetamine) with placebo. The other two studies compared mirtazepine (a noradrenergic and serotonergic antidepressant) with placebo. Neither medication reduced withdrawal symptoms or craving. Amineptine had some limited benefits in increasing adherence to treatment but it has subsequently been withdrawn from the market due to reports of its abuse liability. Shoptaw and colleagues conclude that there are currently no available medications that have been shown to be effective for the treatment of amphetamine withdrawal. It seems unlikely that these conclusions will have any impact on current clinical approaches to the treatment and management of amphetamine withdrawal. I would have been very interested to have read a review on the same topic by these authors which made better use of their expert knowledge and without the constraints imposed on them by the Cochrane methodology.

\section{Michael Gossop, PhD}

National Addiction Centre, Kings College London, UK

Competing interests: None. 